Gut, 1965, 6, 515

\title{
Crohn's disease of the anal region
}

\author{
B. K. GRAY, H. E. LOCKHART-MUMMERY, AND B. C. MORSON
}

From the Research Department, St. Mark's Hospital, London

EDITORIAL SYNOPSIS This paper records for the first time the clinico-pathological picture of Crohn's disease affecting the anal canal. It has long been recognized that anal lesions may precede intestinal Crohn's disease, often by some years, but the specific characteristics of the lesion have not hitherto been described. The differential diagnosis is discussed in detail.

In a previous report from this hospital (Morson and Lockhart-Mummery, 1959) the clinical features and pathology of the anal lesions of Crohn's disease were described. In that paper reference was made to several patients with anal fissures or fistulae, biopsy of which showed a sarcoid reaction, but in whom there was no clinical or radiological evidence of intra-abdominal Crohn's disease. The opinion was expressed that some of these patients might later prove to have intestinal pathology. This present contribution is a follow-up of these cases as well as of others seen subsequently.

Involvement of the anus in Crohn's disease has been seen at this hospital in three different ways:

1 Patients who presented with symptoms of intestinal Crohn's disease who, at the same time, also had disease of the anus in the form of ulceration, fistulae, or fissure-in-ano. These patients were referred because of their intestinal symptoms, the involvement of the anus being an associated finding.

2 Patients referred to the hospital because of symptoms of anal disease but who were found also to have intestinal pathology on clinical or radiological investigation at that time. In this group the symptoms caused by the anal disease overshadowed those due to intestinal involvement or else the latter caused no symptoms.

3 Patients who presented with anal lesions which suggested Crohn's disease both clinically and histologically, who had at the time neither clinical nor radiological evidence of intestinal pathology.

The object of this paper is to present the clinical features, pathology, and subsequent course of this last group of patients.

\section{CLINICAL FEATURES}

There were 19 patients with anal lesions in whom a sarcoid reaction was found on biopsy. The clinical types of anal lesion when the patients were first seen were as follows:

Anal fistula, single or multiple $\ldots \ldots \ldots \ldots 13$

Anal fissures .....................

Anal fissure and fistula.............

Total

The types of fistula included both low level and complex high level varieties. The majority had the clinical features described previously (Morson and Lockhart-Mummery, 1959; Lockhart-Mummery and Morson, 1964) which suggest Crohn's disease, that is, the lesions had an indolent appearance with irregular undermined edges and absence of induraation. They were surprisingly painless. Oedema and a watery discharge were other features. In a few patients the clinical appearances could not be distinguished from the average fissure or fistula due to a non-specific infection; the diagnosis of Crohn's disease in these cases was suggested only by the presence of a sarcoid reaction in biopsy material.

Five of the patients gave a history of recurrent anal lesions requiring surgery over years, before their first attendance at St. Mark's. Three of these presented with unhealed anal wounds which were indolent and remarkably painless.

\section{HISTOLOGY}

Figures 1, 4, and 6 all show sarcoid granulomata, as seen in this site.

Pieces of tissue for histological examination were taken from all 19 patients at the time of operation for the anal lesion. In every case the anal tissues contained a sarcoid reaction consistent with a diagnosis of Crohn's disease or tuberculosis. The histological sections have been reviewed and in only one case is there any reason to question the original 
opinion. It is possible that this one patient had a foreign-body giant cell reaction in his tissues rather than a sarcoid or tuberculoid response. He has remained well for seven years since his operation.

A 'sarcoid reaction' is a purely descriptive term applied to collections of epithelioid cells and giant cells of the Langhans type without any central caseation. In the cases reported here the sarcoid foci always contained epithelioid cells, but occasionally giant cells were absent or very poorly developed. The numbers of lymphocytes and plasma cells within and surrounding the foci were also very variable, and it was exceptional for these to be completely absent. The size of these sarcoid granulomata varied from less than a dozen epithelioid cells with or without one or two giant cells to larger lesions containing many dozens of epithelioid cells. The anal tissues also contained chronic inflammatory changes, abscess formation, and fibrosis of a non-specific character. The sarcoid granulomata were in some patients very sparse but in others relatively large pieces of tissue from the anal region, usually the perianal skin, were riddled with lesions. They were found beneath the epidermis, deep in the dermis, subcutaneous fat and muscle or in the granulation tissue lining the fistulous track, ulcer, or abscess cavity.

The chances of finding sarcoid granulomata in biopsy material from an anal lesion to some extent depend on the amount of tissue available for examination. For this reason small biopsies are usually inadequate and it is best to have larger pieces of tissue removed at the time of operation, at which most or all of the track or lesion can be excised.

A confident distinction between a sarcoid reaction due to Crohn's disease, sarcoidosis, or tuberculosis is impossible on histopathological evidence alone (Jones-Williams, 1964). The presence of caseation in the centre of a focus of epithelioid cells and giant cells is suggestive of tuberculosis, but it is still essential to confirm the diagnosis by demonstrating the tubercle bacillus in histological sections or by cultural methods. It is rarely possible to find bacilli in non-caseating tuberculosis by Ziehl-Neelsen staining of histological preparations of ano-rectal lesions. In the present series of cases attempts to demonstrate tubercle bacilli in histological preparations have invariably failed. A few had guinea-pig inoculation of fresh tissue from the anal lesion, but these were reported as negative.

Giant cells of the foreign-body type are commonly seen in tissue removed from anal fistulae. They usually appear spherical with a tendency for their many nuclei to be centrally placed, in contrast to the peripheral position of the nuclei in Langhans giant cells. They are isolated or in clumps of a few. The giant cells may be placed around foreign matter, such as vegetable material derived from the faeces. However, many seem to be formed from striated muscle cells which have been broken up and destroyed by inflammation and fibrosis. The appearances are reminiscent of the giant cells derived from muscle in myocardial fibrosis. Epithelioid cells are not a feature of an ordinary foreign-body reaction. Their absence is a useful, if arbitrary, distinction from the sarcoid type of tissue response.

\section{FOLLOW-UP}

Thirteen of the 19 patients have remained well since their discharge from hospital for periods varying from four to eight years. Their anal lesions have not recurred and there is no clinical or radiological evidence of intestinal disease. One patient died from a carcinoma of the stomach two months after treatment for her anal lesion.

The remaining six patients have developed intestinal Crohn's disease at intervals varying from 17 months to six years from the first appearance of the anal lesion (Table I). One of these has died as a result of her intestinal disease. The clinical histories and pathology of these six patients are now described. 


\section{ILLUSTRATIVE CASES}

CASE 1 A boy, aged 13 at his first attendance, gave two months' history of bleeding from the anus at stool, painful swollen tags at the anal margin, and some watery discharge from the anus. He had no alteration of bowel habit and no symptoms suggesting any abdominal disorder and his general health was excellent.

Local examination of the anal region revealed two oedematous tags dorsally, each associated with a rather indolent fissure with undermined edges. There was no palpable induration but digital examination was painful. Sigmoidoscopy showed normal rectal mucosa to $25 \mathrm{~cm}$. and proctoscopy did not show any associated papillae or haemorrhoids. A barium meal and follow-through was carried out which showed no abnormality.

The fissures were excised and the oedematous tags removed. Histological examination of the removed tissue revealed a sarcoid reaction suggestive of Crohn's disease (Fig. 1). All bacteriological investigations for tuberculosis were subsequently negative and a Mantoux test at a dilution of 1 in 10,000 was also negative. Healing

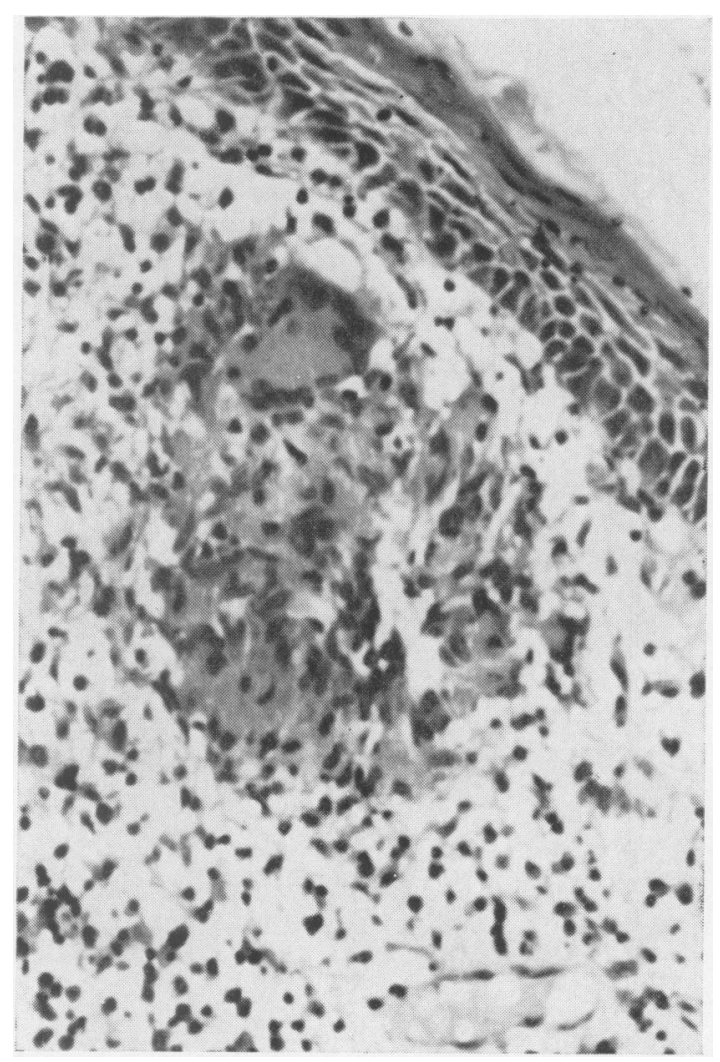

FIG. 1. Case 1. Sarcoid granuloma lying beneath the squamous mucus membrane of the anal canal. It is composed of epithelioid cells with a sprinkling of small round cells and a single giant cell of Langhans type. Haematoxylin and eosin $\times 500$. was extremely slow and required 16 months in all, during which period he had a further excision of the unhealed wounds, but healing eventually occurred after 18 months, when he was discharged from attendance at the hospital with the anal region soundly healed and with no symptoms.

The patient remained perfectly well for a further two years when, now aged 17, he again attended the hospital with a three months' history of right-sided abdominal colic, diarrhoea up to four times daily, loss of weight of over a stone in two months, anorexia, and lassitude. Examination at this time revealed some localized tenderness in the right iliac fossa but no other abdominal signs and the anal region remained well healed, and sigmoidoscopy was still perfectly normal. A further barium meal and follow-through was carried out which revealed a stricture of the terminal ileum compatible with a diagnosis of Crohn's disease. Laparotomy showed Crohn's disease of the terminal ileum. There were some skip lesions in the ileum and also a 3 in. long segment of disease in the transverse colon. An exclusion operation was performed with end-to-side anastomosis of the ileum to the distal transverse colon. A biopsy of the enlarged glands in the root of the ileal mesentery showed a sarcoid reaction consistent with Crohn's disease. Post-operatively he made an uneventful recovery but diarrhoea persisted though somewhat improved.

He was re-admitted again one year after operation with symptoms and signs suggestive of subacute obstruction. Laparotomy was performed at which there was no evidence of active Crohn's disease but a loop of jejunum was adherent to the previous anastomosis and was freed. The disease in the terminal ileum and colon appeared quiescent but a right hemi-colectomy was carried out. The patient made an uneventful recovery and has remained well since.

Pathology Examination of the surgical specimen showed thickening of the bowel wall, scarring and stricture formation in both the ileum and transverse colon (Figs. 2 and 3). There was no mucosal ulceration. Microscopically there was patchy hyperplasia of lymphadenoid tissue in diseased areas with scattered sarcoid foci in both the bowel wall and regional glands. Many of these appeared to be undergoing a process of hyalinization suggesting healing and resolution of the active disease observed a year previously.

CASE 2 A boy, aged 16 at his first attendance, gave a history of anal fistula diagnosed 14 months previously. Three operations were performed at another hospital, but the wound did not heal. He had no symptoms apart from anal discomfort, and general physical examination was negative.

Local examination of the anal region revealed an unhealed wound at the posterior anal margin. This presented an indolent appearance with undermined edges and exuberant granulations. The wound extended throughout the length of the previous operation for a high fistula, which had opened dorsally into the anal canal just below the upper third of the anal sphincters. The wound was not indurated and was remarkably pain-free. Sigmoidoscopy showed normal appearances of the rectal mucosa to $25 \mathrm{~cm}$. 


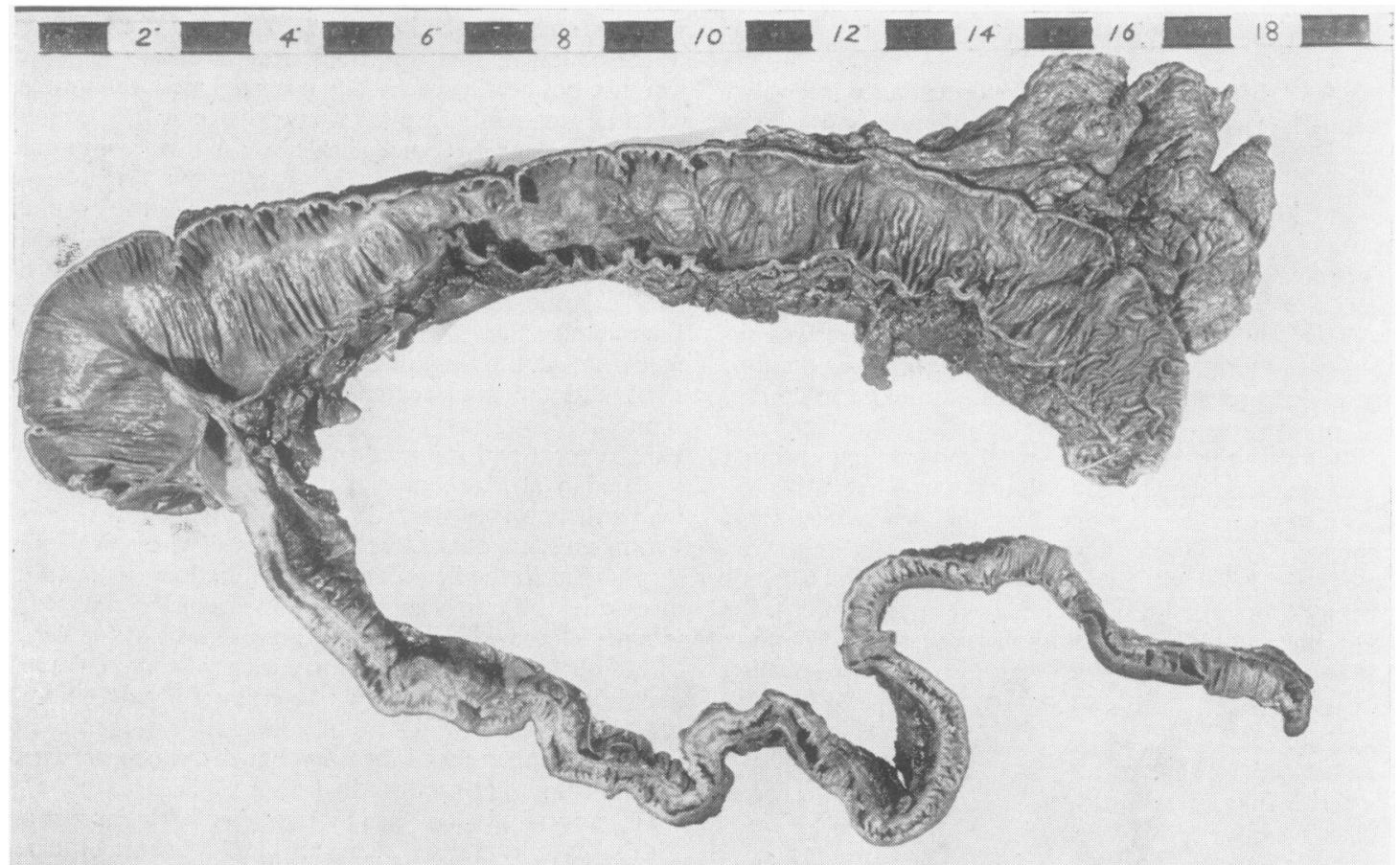

FIG. 2. Case 1. There is fibrous stenosis of the terminal ileum. The ascending and transverse colon shows longitudinal scarring of its mucosal surface. There is no ulceration.

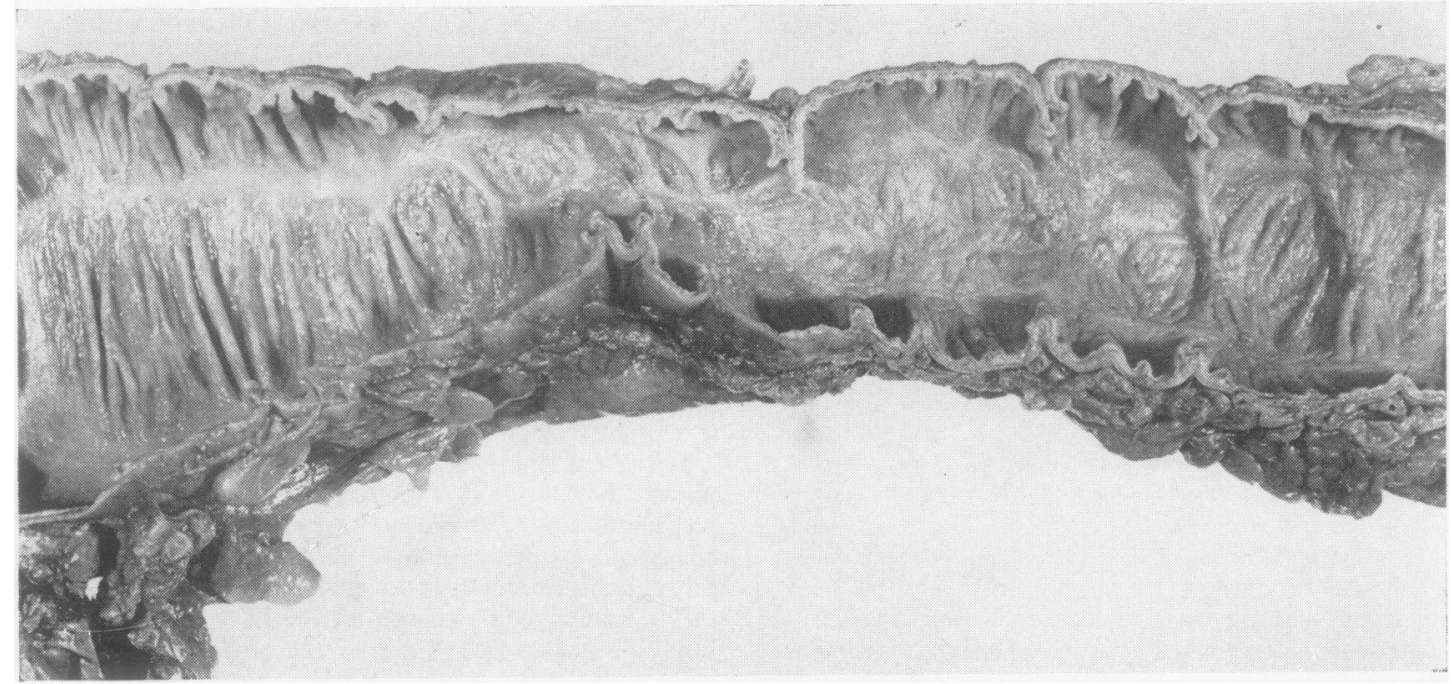

FIG. 3. Case 1. Close-up of the transverse colon in Fig. 2 to show the extensive scarring caused by submucosal inflammation and fibrosis. 
The wound was gently trimmed and material sent for histological examination. This confirmed the presence of a sarcoid reaction in granulation tissue, suggesting a diagnosis of Crohn's disease. All bacteriological investigations for tuberculosis were negative. A barium meal and follow-through showed no evidence of intestinal disease. The wound healed very slowly following operation but in 11 months it was completely healed.

This patient remained perfectly well for four years, when at the age of 20 , he complained of abdominal colic after meals, vomiting, diarrhoea, up to three or four loose water stools daily, and loss of weight. There was some tenderness in the right iliac fossa but the anal region was perfectly healed and sigmoidoscopy was again normal. Laparotomy was performed and the terminal ileum and caecum were found to be the site of extensive Crohn's disease. A right hemi-colectomy was carried out with end-to-end anastomosis. The patient made an uneventful recovery and has remained well since.

Pathology Examination of the operation specimen showed changes typical of Crohn's disease of the terminal ileum including scattered sarcoid granulomata.

CASE 3 A man, aged 24 at his first attendance, gave an 18-month history of anal pain on defaecation, watery and purulent discharge from the anal region, and some perianal irritation. He had two previous operations for a fistula-in-ano, five and 13 months before his attendance at St. Mark's Hospital, but without complete healing. He had no abdominal symptoms or bowel disorder, his general health was excellent, and general physical examination was negative.

Examination of the anal region revealed an anterior anal fistula with an associated anterior fissure. Sigmoidoscopy to $25 \mathrm{~cm}$. was negative.

Examination under anaesthesia revealed a complex high anal fistula with four separate openings into the anal canal and lower rectum. These were laid open and the granulating area was skin-grafted two weeks later. The graft took well and healing was rapid, and the patient was discharged six weeks after operation with his wound well healed. Histological examination of the excised tissue revealed a sarcoid reaction suggestive of Crohn's disease (Fig. 4). All bacteriological investigations for tuberculosis were negative but a Mantoux test was positive at 1 in 10,000 dilution. No $x$-ray studies were carried out at that time.

The patient remained perfectly well for two years when he re-attended, complaining now of some intestinal colic, mild abdominal distension, and anorexia, these symptoms having been present for two or three months. His bowels were regular and there was no complaint of diarrhoea. There were no abnormal signs on general physical examination and the anal region remained well healed. A barium follow-through examination revealed gross irregularity of the terminal ileum and caecum suggestive of Crohn's disease.

Laparotomy was performed which revealed an area of Crohn's disease involving the terminal $20 \mathrm{~cm}$. of ileum with a mass of adhesions to the caecum. A right hemicolectomy was carried out with end-to-end ileo-transverse

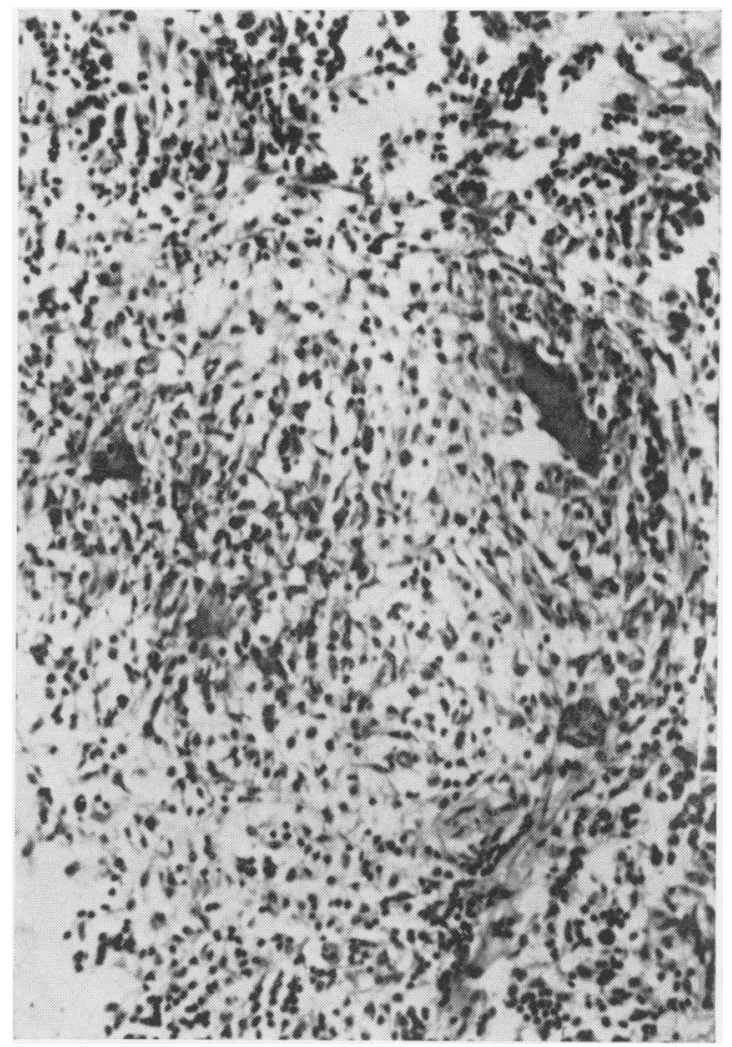

FIG. 4. Case 3. Sarcoid granuloma in ano-rectal tissues. Collections of epithelioid cells and many round cells are present with three Langhans giant cells. Haematoxylin and eosin $\times 500$.

anastomosis. The patient made an uneventful recovery and has remained well since.

Pathology The macroscopic and microscopic appearances of the surgical specimen showed typical Crohn's disease of the terminal ileum (Fig. 5). The tissues of the bowel wall and the regional lymphatic glands contained scattered sarcoid foci.

The mucosal ulceration in the specimen becomes less extensive from the ileo-caecal valve towards the proximal limit of excision when there are occasional tiny 'aphthoid' ulcers in the mucosa. These cannot be seen in the photograph. Histologically they proved to be ulcerating lymphoid follicles.

CASE 4 A woman, aged 37 at her first attendance, gave a short history of bleeding and discharge of pus from the anal region with pain at the anus on defaecation and some perianal irritation. Her general health was excellent and general physical examination revealed noabnormality.

Examination of the anal region showed a posterior anal fistula with associated oedematous skin tags. Sigmoidoscopy was normal to $25 \mathrm{~cm}$. A barium meal and follow-through was carried out but no abnormality was seen anywhere in the small or large bowel. 


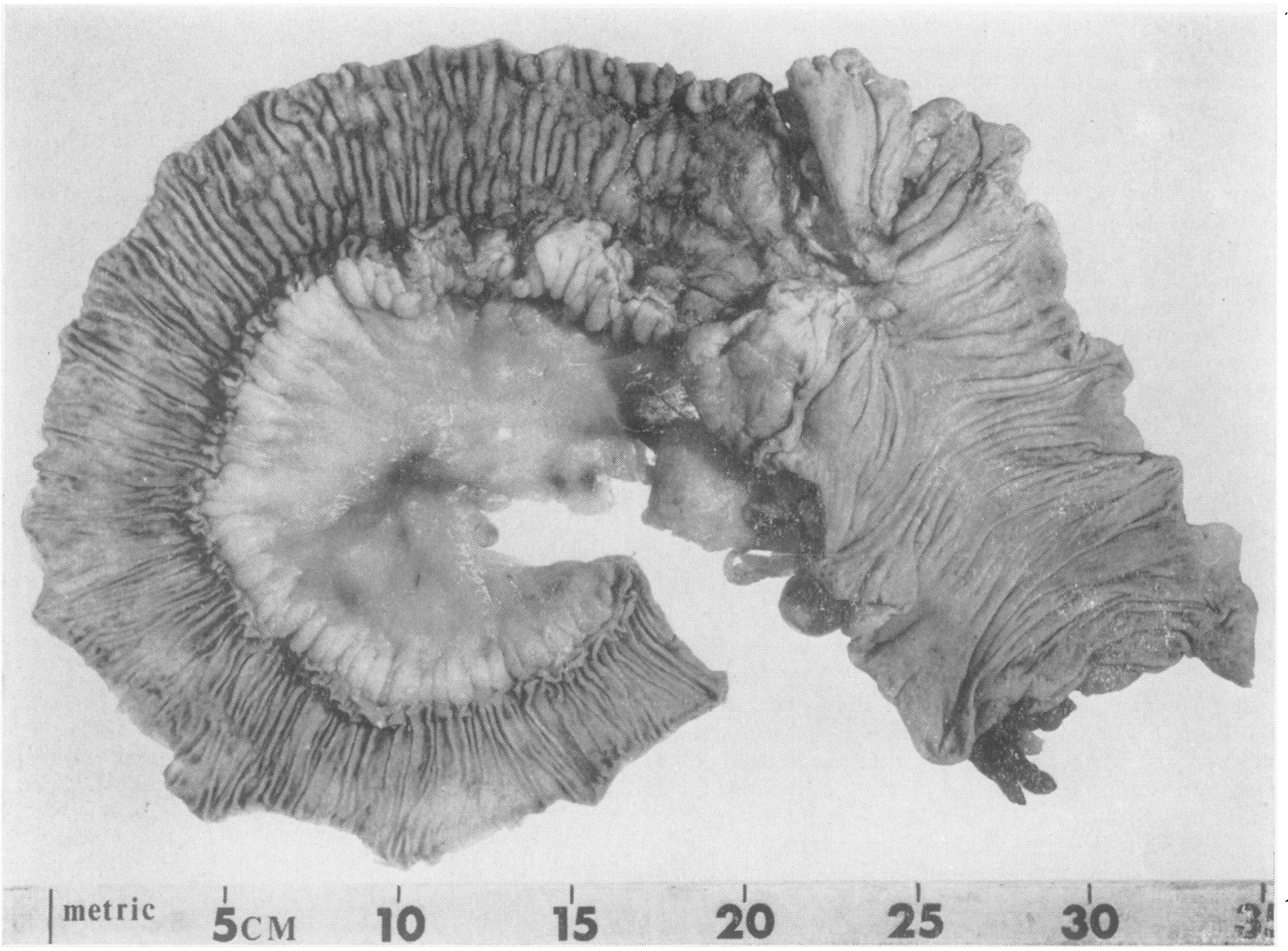

FIG. 5. Case 3. Right hemicolectomy specimen for Crohn's disease of the terminal ileum. There is patchy mucosal ulceration becoming more extensive from the proximal part of the specimen towards the ileo-caecal valve. The latter shows considerable stenosis.

The patient was admitted six months later and the fistulous track was laid open. Tissue sent for histological examination revealed a sarcoid reaction suggestive of Crohn's disease. All bacteriological investigations for tuberculosis were negative. A further barium meal carried out at this time was reported as perfectly normal. Healing of the anal region was slow but almost complete apart from one small area at the anal verge 15 months after the original operation.

She then began to complain of colicky abdominal pain, loss of weight, and that the diarrhoea was now worse, with seven loose watery motions daily. A barium followthrough at this time revealed changes in the terminal ileum compatible with a diagnosis of Crohn's disease. Faecal fat estimations showed a moderate steatorrhoea. A laparotomy was performed and a 4-foot segment of terminal ileum was found to be involved by Crohn's disease showing numerous skip lesions. An exclusion operation was performed with end-to-side anastomosis of the ileum to the transverse colon. The patient's postoperative recovery was uneventful and she remains well with the anal region satisfactorily healed and her general health excellent. The time interval from her first symptoms of anal disease to the recognition of intestinal pathology was 17 months.

CASE 5 A woman, aged 39 at her first attendance at St. Mark's Hospital, gave a six-month history of bleeding and discharge from the anal region, pain in the anus on defaecation, and a palpable swelling at the anal margin. General physical examination showed no abnormality.

Examination of the anal region showed oedematous tags posteriorly with a midline dorsal fistula-in-ano. There was a discharge of watery pus from the latter and there was a considerable induration of the posterior wall of the anal canal. Sigmoidoscopy was normal to $25 \mathrm{~cm}$. Barium follow-through studies showed no evidence of any intestinal lesion.

The patient was admitted and the fistulous track laid open. Tissue sent for histological examination showed a florid sarcoid reaction suggestive of Crohn's disease (Fig. 6). All bacteriological investigations for tuberculosis were negative and the Mantoux test was negative at all dilutions. Healing of the anal region was slow but was complete in 11 months.

The patient re-attended 15 months later, that is 22 


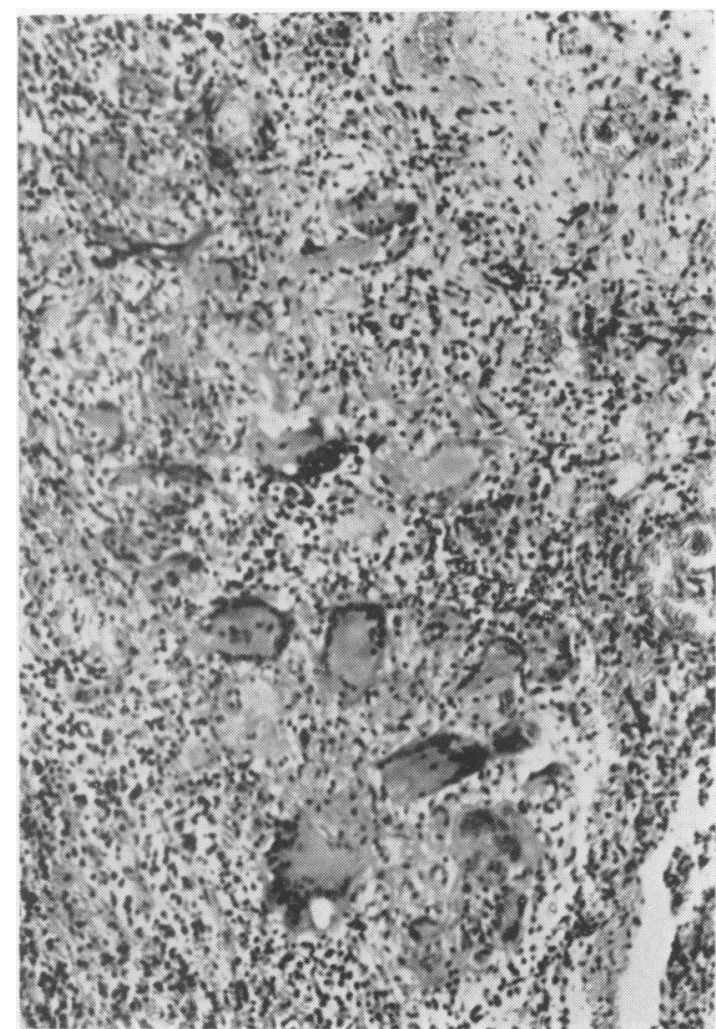

FIG. 6

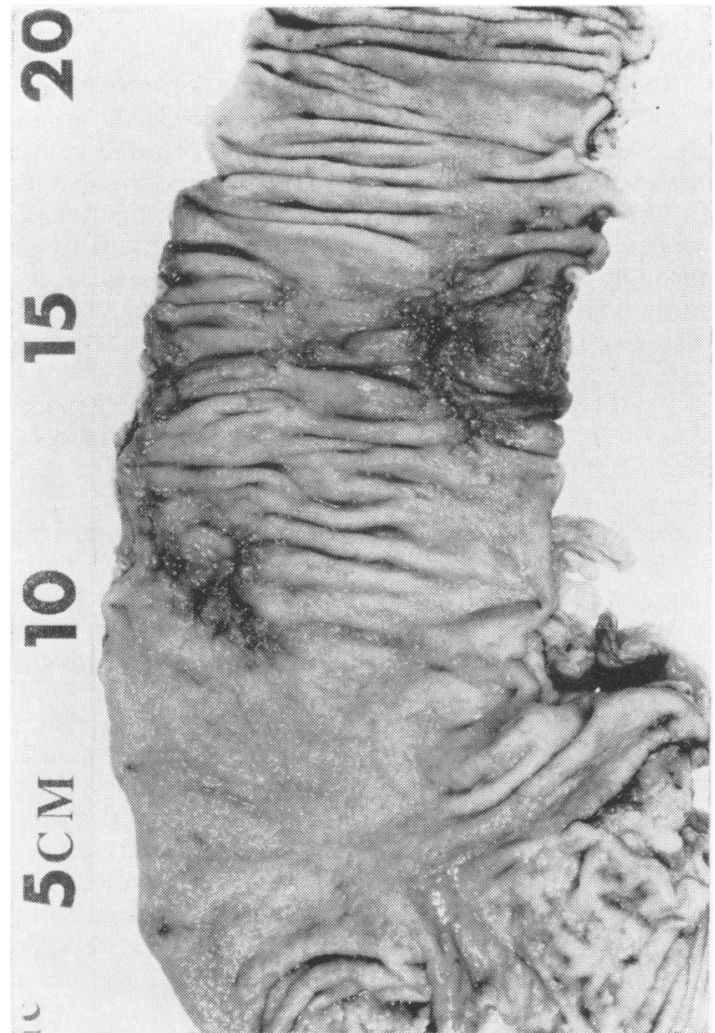

FIG. 7.

FIG. 6. Case 5. Sarcoid granulomata in ano-rectal tissues. There is infiltration with chronic inflammatory cells around epithelioid cells and an unusually large number of Langhans giant sells. Haematoxylin and eosin $\times 200$.

FIG. 7. Case 5. Close-up of extended right hemicolectomy specimen showing two irregular areas of ulceration in the ascending colon and a diffusely granular mucosa.

months after her first symptoms of anal disease, with recent onset of severe right-sided abdominal colic, continuous diarrhoea with the passage of three to five watery stools daily, and anorexia. Barium followthrough studies at this time suggested early Crohn's disease of the right colon. One month later laparotomy showed evidence of Crohn's disease in the ascending colon. A right hemi-colectomy was carried out from which the patient made an uneventful recovery.

Pathology (Fig. 7) The mucosa of the terminal ileum appeared slightly granular. There is one tiny ulcer $2 \mathrm{~cm}$. proximal to the ileocaecal valve. The appendix appears normal. In the caecum and ascending colon the mucosa appears granular. In the ascending colon $5 \mathrm{~cm}$. above the ileocaecal valve there is an area of induration and ulceration about $2 \mathrm{~cm}$. in diameter. Another area of induration and ulceration lies $10 \mathrm{~cm}$. above the ileocaecal valve. This is $3 \mathrm{~cm}$ in diameter. and the mucous membrane between it and the first area mentioned shows a few small patches of superficial ulceration. The bowel distal to these frankly diseased areas appears normal. The regional lymphatic glands are not enlarged.
Histology The diseased areas in the ascending colon show patchy mucosal ulceration with hyperplasia of lymphadenoid tissue extending deep into the submucosa which is also widened by oedema. There is little penetration of the deep muscle coats by the inflammatory changes. There are abscesses in the submucosa and scattered sarcoid granulomata. The mucosa of the terminal ileum and caecum shows mild chronic inflammatory changes. Some mucosal lymphoid follicles appear to be undergoing early abscess formation. The regionally $\mathrm{mph}$ glands are normal.

CASE 6 A woman, aged 36, gave a four-month history of pain in the anal region on defaecation, associated with some bleeding and the discharge of watery pus from the anus. She had no abdominal symptoms and no alteration of bowel habit and her general health was good. General physical examination was negative.

Examination of the anal region revealed a posterior fistula-in-ano of the low-level variety, associated with a dorsal fissure and rather oedematous skin tags. The region presented rather an indolent appearance and there was 
little induration. Sigmoidoscopy revealed normal rectal mucosa to $25 \mathrm{~cm}$.

The patient was admitted for operation at which the fistulous track was laid open and the oedematous tags were excised. The histological report on the excised tissue showed the presence of a sarcoid reaction suggestive of Crohn's disease. All bacteriological investigations for tuberculosis were negative. A barium meal and followthrough was carried out but the bowel appeared quite normal. Following operation the healing of the wound was extremely slow and the patient failed to return for follow-up examination.

However, she apparently remained well for 12 months when she began to complain of abdominal colic, anorexia and diarrhoea, with the passage of two to three stools daily. She was admitted to another hospital and a laparotomy was performed at which the terminal 12 in. of ileum and the caecum were found to show typical changes of Crohn's disease. It was not considered desirable to perform a resection and the abdomen was closed. Recovery was uneventful and she was subsequently discharged.

However, a few months later she was re-admitted to the same hospital with a large abscess pointing through the laparotomy scar. This was opened and contained a large amount of pus and she subsequently developed a faecal fistula which continued to discharge. A re-exploration of the abdomen was carried out when a mass of Crohn's disease involving the terminal ileum was found with two faecal fistulae to the abdominal wall. An exclusion operation was carried out, the ileum being anastomosed end-to-side with the transverse colon.

The patient remained well and improved following this operation but after a further 15 months she was admitted to yet another hospital with marked deterioration in her condition from which she subsequently died.

\section{DISCUSSION}

Crohn's disease of the anus can present either as an ulcerative condition, usually in continuity with disease of the rectum, or in the form of an anal fistula or fissure. Although such fissures or fistulae may show clinical characteristics suggestive of Crohn's disease the appearances are sometimes indistinguishable from those due to a non-specific infection. For this reason it is always important that tissue should be removed for histological examination from anal fissures or fistulae at the time of surgical treatment (Gabriel, 1921). The detection of a sarcoid reaction suggests a diagnosis of Crohn's disease but it remains important to exclude tuberculosis as a cause for the appearances by appropriate clinical, radiological, and laboratory tests.

A sarcoid reaction is found in a great variety of conditions throughout the tissues of the body of which the most familiar is the clinico-pathological syndrome known as sarcoidosis. However, involvement of the gastro-intestinal tract in this disease is extremely uncommon. For all practical purposes it can be ruled out as a cause of the sarcoid response in the anal lesions described here. In the intestines and anal region the most likely causes for a sarcoid reaction are either Crohn's disease or tuberculosis, although it may exceptionally occur as a tissue response to local malignant disease.

The presence of a sarcoid reaction in anal tissues should be followed up by persistent attempts to exclude tuberculosis. In the first place a Mantoux test should be performed, for if this is negative to a dilution of 1 in 100 tuberculin a diagnosis of tuberculosis is for all practical purposes ruled out when dealing with chronic disease. On the other hand, if the Mantoux test is positive it is advisable to obtain fresh tissue from the anal lesion for guinea-pig inoculation. This may have to be repeated if negative. A chest radiograph is important, for ano-rectal tuberculosis is uncommon in patients without pulmonary disease. Despite vigorous attempts to prove tuberculosis as a cause for the sarcoid reaction in ano-rectal tissue it is sometimes impossible to make a clear decision although the clinical history and follow-up information, as reported here, will indicate which is the more likely diagnosis. In the experience of this hospital Crohn's disease is a much more common cause of a sarcoid reaction in anal lesions than tuberculosis.

A sarcoid or tuberculoid reaction is present in only about $60 \%$ of all cases of Crohn's disease. It follows that the diagnosis of Crohn's disease of the anus, whatever the clinical type of anal lesion, can only be made on histological examination in a similar proportion of cases. In those patients without a sarcoid reaction the diagnosis of anal Crohn's disease rests on the clinical appearance of the anal lesion together with any other evidence of intestinal involvement. It is probable that some anal lesions due to Crohn's disease remain unrecognized for lack of characteristic clinical or histological features to distinguish them from a simple non-specific type of infection. This explains why patients with Crohn's disease of the small or large bowel often have a history of an anal lesion many years previously which has responded, if reluctantly, to local surgical treatment, but had not been recognized originally as due to Crohn's disease. Apart from lack of the characteristic clinical and histological features, the failure to biopsy all anal lesions at the time of surgical treatment must be a frequent cause of failure to recognize the pathology. The anal lesions of Crohn's disease are not secondary to the intestinal involvement but a local manifestation of a disease which also involves single or multiple segments of any part of the gastrointestinal tract from the stomach to the anus inclusive.

It is probable that Crohn's disease is prone to 
affect the anus because of the aggregation of lymphoid tissue around the anal glands (Parks and Morson, 1962) in the same way that Crohn's disease most commonly affects the terminal ileum because of the great amount of lymphoid tissue in this part of the gut.

Follow-up of the 19 patients with suspected Crohn's disease of the anus has so far revealed six who have developed typical intestinal disease which was not detectable at the time of diagnosis of the anal lesion. The time interval between the onset of anal symptoms to the diagnosis of intestinal disease varied from 17 months to over five years (Table I). It is probable that further patients in this series will develop intestinal pathology, for cases have been reported in which there has been a history of an anal lesion as much as 14 years before the development of intestinal disease (Crohn and Yarnis, 1958). Certainly it has been the experience of this hospital that patients with typical Crohn's disease of the small or large bowel often have a history of anal fissure or fistula going back many years.

The 13 patients with suspected Crohn's disease of the anus who have not yet developed any abdominal signs or symptoms may have clinically latent intestinal pathology, either in the form of early, active lesions or as 'burnt out' disease. That Crohn's disease can resolve in this way is illustrated in case 1. It is also possible that Crohn's disease can involve the anus alone without any synchronous or subsequent disease higher up the gastrointestinal tract. Such may be the explanation for some anal fissures or fistulae which are reluctant to heal despite correct surgical treatment. On the other hand there is evidence to suggest that Crohn's disease can be a chronic condition which remains clinically latent for many years despite the presence of pathological changes. Late recurrence, as much as 10 or 15 years after right hemicolectomy for classical Crohn's disease of the terminal ileum, is quite common. The incidence of recurrence increases with the length of follow-up (Van Patter, Bargen, Dockerty, Feldman, Mayo, and Waugh, 1954; Cooke, 1955). In the six patients reported here who have developed intestinal 'recurrence' years after the diagnosis of Crohn's disease of the anus it is probable that the intestinal pathology was present at the time of onset of the anal symptoms, although at a clinically and radiologically undetectable stage. The anal lesions of Crohn's disease may be regarded as a signpost to intestinal pathology which may not be manifest until many years after the anal disease has healed.

The long latent period between the onset of anal symptoms and the development of intestinal disease may be at least partly explained by appearances sometimes seen in surgical specimens of Crohn's disease of the small or large bowel. Brooke (1953) described small localized 'aphthoid' ulcers of the mucous membrane at some distance from more obviously diseased areas of gut. Such tiny ulcers were particularly prominent in one of the cases reported here (case 3) and they have been observed in specimens resected at this hospital on many other occasions. Histologically they are seen to be due to ulceration of small lymphoid follicles in the mucosa. They are sometimes detected close to the limit of excision of resected segments of gut and may well be the pathological basis for late recurrences after surgical treatment. In the cases studied here it is possible that such areas of early ulceration were already present in the mucosa of the gut at the time of onset of symptoms due to anal disease but that they have taken years to develop to a stage where they became radiologically or clinically detectable. It is likely that disease of the anus sometimes presents at an earlier stage than the intestinal pathology, not only because it leads to symptoms, but because of accessibility to examination.

If, as we suspect from our experience of these cases, intestinal Crohn's disease can sometimes be diagnosed by biopsy of an anal lesion, before any abdominal symptoms have become manifest, it remains to be seen whether such early diagnosis could be of real benefit to the patient. But it is possible that treatment (with sulphasalazine or steroids, for example) given at this stage might suppress the overt development of the intestinal lesion, or possibly ameliorate its course.

\section{SUMMARY}

The clinical features, biopsy appearances, and subsequent history of 19 patients with suspected Crohn's disease of the anus, who had no clinical or radiological evidence of intestinal pathology at the time of diagnosis, have been described. Up to the present time six of these have developed intestinal Crohn's disease after a time interval varying from 17 months to over five years after the onset of anal symptoms. The clinical histories and pathology of these cases have been described and illustrated. It is possible that intestinal 'recurrences' may yet take place among the remaining 13 patients. The diagnosis of Crohn's disease of the anus is discussed as well as the possible explanation for the long latent period between the onset of symptoms due to anal disease and the development of symptoms caused by the intestinal pathology.

We would like to thank the consultant staff of St. Mark's Hospital for permission to study their cases, in particular Mr. Henry Thompson and Mr. A. G. Parks, also Mr. 
Colin Craig, F.R.C.S., of Lowestoft. The photographs were taken by Mr. Norman Mackie. Mr. H. J. R. Bussey and Mr. Lloyd Soodeen gave valuable assistance. The expenses of this research were partly defrayed out of a grant to the Research Department of St. Mark's Hospital from the Clinical Research Committee of the Hammersmith and St. Mark's group of hospitals.

\section{REFERENCES}

Brooke, B. N. (1953). What is ulcerative colitis? Lancet, 1, 1220-1225. Cooke, W. T. (1955). Nutritional and metabolic factors in the aetiology and treatment of regional ileitis. Ann. roy. Coll. Surg. Engl., 17, 137-158.
Crohn, B. B., and Yarnis, H. (1958). Regional Ileitis, 2nd ed. Grune and Stratton, New York.

Gabriel, W. B. (1921). Results of an experimental and histological investigation into seventy-five cases of rectal fistulae. Proc. roy. Soc. Med., 14, Part III, Section of Surgery, 156-161.

Jones-Williams, W. (1964). Histology of Crohn's syndrome. Gut, 5, 510-516.

Lockhart-Mummery, H. E., and Morson, B. C. (1964). Crohn's disease of the large intestine. Gut, 5, 493-509.

Morson, B. C., and Lockhart-Mummery, H. E. (1959). Anal lesions in Crohn's disease. Lancet, 2, 1122-1123.

Parks, A. G., and Morson, B. C. (1962). The pathogenesis of fistulain-ano. Proc. roy. Soc. Med., 55, 751-754.

Van Patter, W. N., Bargen, J. A., Dockerty, M. B., Feldman, W. H., Mayo, C. W., and Waugh, J. M. (1954). Regional enteritis. Gastroenterology, 26, 347-450. 